Investigaciones Fenomenológicas, n. 13, 2016, 13-30.

e-ISSN: $1885-1088$

\title{
EL MOVIMIENTO DEL MUNDO Y EL PROBLEMA DEL APARECER ${ }^{1}$
}

\section{THE MOVEMENT OF THE WORLD AND THE PROBLEM OF APPEARING}

\author{
Renaud Barbaras \\ Universidad Paris 1
}

\begin{abstract}
Resumen: Este texto presenta en un primer momento el movimiento propio de la fenomenología a-subjetiva de Patočka a partir de su crítica de la versión subjetivista del aparecer, para así posteriormente mostrar una dinámica fenomenológica que comprende la manifestación como la obra misma del mundo. En este sentido, el destino de la fenomenología se trasciende en la elaboración del a priori universal de la correlación que estipula la universalidad de una manifestación anónima. Es así que finalmente la fenomenología se modifica en una cierta cosmología que nos obligará a repensar una nueva acepción del ser del sujeto y que postula un nuevo sentido de ser del mundo a partir de una concepción asubjetiva del aparecer.
\end{abstract}

Palabras clave: Patočka, sujeto, mundo, fenomenología a-subjetiva, cosmología

\begin{abstract}
This text initially presents the specific movement of Patočka's a-subjective phenomenology, itself based on his critique of the subjectivist version of the Appearing. Subsequently a phenomenological dynamic comes to light, which understands the manifestation as the very world's work. In this way, the phenomenological's fate is inflected by the formulation of the universal a priori of correlation that stipulates the universality of an anonymous manifestation. Finally, phenomenology is perceived as a certain cosmology which will commit us to rethink of a new meaning for the subject's being and of the world's being from an asubjective's conception of the Appearing.
\end{abstract}

Keywords: Patočka, subject, world, asubjective phenomenology, cosmology

\footnotetext{
${ }^{1}$ Artículo original: Barbaras, R. (2013/3), "Le mouvement du monde et le problème de l'apparaître", en Philosophie, Editions de Minuit, $\mathrm{N}^{\circ} 118$, p. 21-33. Agradecemos a la revista Philosophie, dirigida por Dominique Pradelle, y sobretodo a Renaud Barbaras, por la gentileza de haber permitido la traducción presente.
} 
Este artículo pretende en primer lugar restituir sistemáticamente el movimiento propio del pensamiento de Patočka, movimiento que no corresponde a ninguna exposición cronológica y que no ha sido expuesto en ninguna parte bajo esta forma, pero que, a mi modo de ver, subyace a aquello que Patočka denominó fenomenología a-subjetiva. Este movimiento toma su punto de partida en una crítica de la versión subjetivista del aparecer, principalmente husserliana, dando lugar a aquello que denominaré una dinámica fenomenológica. Dicha dinámica comprende la manifestación como la obra misma del mundo y conduce en tal caso a realizar la distinción entre una manifestación primaria o anónima y una manifestación secundaria, aquella que corresponde propiamente tal a la manifestación como donación a un sujeto. Pero mi artículo trae consigo asimismo una vertiente crítica, que se encuentra, en cierto modo, aludida por la propia curva del pensamiento de Patočka. De hecho, reconocer una manifestación primaria anónima a contrapelo de la manifestación subjetiva secundaria, nos expone al inquietante problema del pasaje del uno al otro, es decir al fondo mismo de la razón subjetiva. Ahora bien, de ello depende el sentido y el destino mismo de la fenomenología, en la medida en que ésta tiene como tarea la de elaborar el a priori universal de la correlación, a priori que estipula que todo ente trascendente reviste por esencia una relación a unos modos subjetivos de donación. Entonces, ¿cómo conciliar la universalidad de dicho a priori con el descubrimiento de una manifestación anónima que no tiene como sujeto sino el mundo mismo? ¿Cómo integrar la dimensión propiamente subjetiva del aparecer en una perspectiva para la cual el parecer es la obra misma del mundo? Nosotros mostraremos que Patočka se sitúa en un marco que no le permite resolver esta cuestión. Sin embargo, por la misma razón seremos conducidos a preguntarnos por las condiciones que permitirán resolver dicho problema, o más bien a mostrar que su consideración arrastra una cierta modificación de la fenomenología en dirección de una metafísica, ella misma entendida en un sentido bastante singular. Esta modificación dará lugar ella misma a una nueva acepción del sentido de ser del sujeto que ha faltado en Patočka, a pesar de que ella asoma en ocasiones. En el fondo, nuestro planteamiento consiste en explorar hasta sus últimas consecuencias la crítica patočkiana del subjetivismo, lo cual significa reconocer que él mismo no se libera del todo de esta crítica.

La fenomenología patočkiana no pretende ser otra cosa que una tentativa de llevar hasta el fin el planteamiento husserliano, en la medida en que éste 
continúa estando caracterizado por una cierta inconsecuencia o, al menos, por una forma de imperfección [inaboutissement]. Dicha inconsecuencia se puede resumir por la desatención respecto de la autonomía del aparecer, que procede ella misma de una comprensión insuficientemente radical de la epojé fenomenológica. En efecto, pasando desde la epojé hacia una reducción entendida como reconducción a la región conciencia, a la esfera de datos inmanentes, Husserl traiciona la originalidad y la auto-suficiencia del campo fenomenal ya que lo hace basarse en un ente real, que tiene como único privilegio la adecuación en la auto-donación, es decir en el fondo la transparencia a sí mismo. Como lo escribe Patočka, "la subjetividad, como la inmanencia a la cual se recurre en última instancia, no se vuelve posible sino por la escisión de la esfera fenomenal en tanto tal, escisión que se produce porque no vemos cómo pensar la esfera fenomenal en tanto que algo autónomo, porque así nos creemos entonces con la obligación de sustentarla a partir de algo procedente del ámbito de lo real (las "aprehensiones", los "actos idénticos", etc.). Esta realidad, inicialmente modesta, hace una carrera prodigiosa, pasando de ser un ente en el mundo que ella era sin más, a la sustancia de la constitución misma del mundo". ${ }^{2}$ La escisión de la esfera fenomenal de la que habla Patočka se refiere a un desdoblamiento del sentido de lo subjetivo: mientras que la subjetividad no puede significar nada más que la naturaleza del campo fenomenal trascendente, sin embargo en su calidad de relativa, en su trascendencia misma, a un sujeto viviente, ella llega a designar una cierta esfera de ser, aquella de las vivencias, con lo que se da lugar a la distinción entre lo subjetivo como vivencia y lo fenomenal que aparece en la vivencia y que, en verdad, es constituido en ella. Es en ese movimiento, perfectamente identificado por Patočka, que se resume aquello que podríamos denominar la subjetivación del campo fenomenal. Por cierto, dicho movimiento descansa él mismo en última instancia sobre el privilegio otorgado a la vivencia, privilegio que no hace referencia sino a su modo de aparecer y por lo tanto continúa siendo del orden del conocer: la vivencia posee en efecto como propio aquello de darse a sí misma sin distancia ni resto, en una plenitud de presencia que, desde el punto de vista de Husserl, y en consonancia con Descartes y Brentano, es la medida misma de aquello que es verdadera-

\footnotetext{
2 Patočka, J. (1988). Qu'est-ce que la phénoménologie ?, trad.fr. E. Abrams, Grenoble, J. Millon, p. 209 [cabe señalar que realizo una traducción propia de dicha referencia, que aparece en francés en el texto original. Para las futuras referencias, remitirse al texto citado en francés].
} 
mente. También podríamos decir que el lugar de excepción otorgado a la esfera de inmanencia descansa en última instancia sobre un intuicionismo que Husserl no discutirá jamás. Como lo dice Patočka, "La certeza de sí de la existencia del ego, del sum, es interpretada como presencia, la presencia como autodonación originaria. Ahora bien, la autodonación originaria requiere un objeto correspondiente". ${ }^{3}$ Dicho objeto no es otra cosa que la vivencia [vécu]. En otras palabras, si la apodícticidad de la existencia del ego - que, desde luego, Patočka no discute- es interpretada a partir del modelo intuitivo como adecuación, entonces debemos plantear un objeto correlativo de aquella intuición y el ego se ve por lo tanto en cierto modo reificado bajo la forma de vivencias inmanentes. Se tratará entonces al contrario para Patočka de distinguir la apodícticidad de la existencia del ego de toda forma de adecuación ("no hay "en" el ego nada que ver como tal" ${ }^{\prime 4}$ ), lo que permitirá evidentemente allanar el camino de una interrogación sobre el sentido de ser del ego.

Como lo habíamos anunciado anteriormente, en este intento de subjetivación del campo fenomenal, Husserl continúa estando prisionero de la actitud natural en un sentido más profundo y desapercibido. De hecho, ésta última no consiste en primera instancia en la tesis de existencia del mundo, o mejor dicho, esta tesis de existencia es una de las formas que adopta el gesto aún mucho más secreto consistente en reconducir el aparecer a un apareciente, cualquiera que sea su naturaleza. La ingenuidad adopta aquí el aspecto de la circularidad puesto que, reconduciendo el aparecer a un cierto apareciente, ya lo presuponemos, nos lo damos en el instante mismo que lo queremos constituir. Esto es exactamente lo que hace Husserl con el método reductivo: reconduce el aparecer desde el campo fenomenal hacia un cierto apareciente singular, que siempre ya ha aparecido, que concentra ya en él mismo la ley del aparecer, a saber, las vivencias inmanentes. Ahora bien, como lo señala Patočka, "hay un campo fenomenal, un ser del fenómeno como tal, que no puede ser reducido a ningún ente que aparece en su seno y que es entonces imposible de explicarlo a partir de lo óntico, sea éste de especie naturalmente objetiva o egológicamente subjetivo". ${ }^{5}$ Así, es lo mismo reconducir el aparecer a un mundo que reposa en sí mismo o a una subjetividad constituída por vivencias puesto que, en

\footnotetext{
${ }^{3}$ Idem, p. 243.

${ }^{4}$ Idem, p. 212.

${ }^{5}$ Idem, p. 239.

Investigaciones Fenomenológicas, n. 13, 2016.
} 
ambos casos, la autonomía y la originalidad del aparecer se encuentran comprometidas. En este sentido, a pesar de las apariencias, el subjetivismo husserliano queda prisionero de una forma recalcitrante de realismo, aquél sostenido por el credo intuicionista. De allí se sigue que la verdadera epojé es aquella que nos conduce al aparecer como tal, en su autonomía, que neutraliza en consecuencia todo apareciente, incluso subjetivo. La única epojé que vale es aquella que incorpora el ego en su propio paréntesis, dándose así los medios para acceder al a priori del que depende el ego, en vez de concebir como a priori al ego mismo. Esto es lo que Patočka nos sugiere: "¿Qué ocurriría si la tesis del sí mismo propio no estuviese sustraída a la epojé, si ésta fuese concebida de manera completamente universal? [...] ¿No sería posible que la inmediatez de la donación del ego sea un "prejuicio" y que la experiencia de sí mismo tenga, al igual que la experiencia de las cosas, un a priori específico que vuelve posible el aparecer del ego?". ${ }^{6}$

Esta epojé radicalizada permite en cierto modo rehacer en sentido inverso el camino recorrido por Husserl y de esta manera restituir su verdad. Ella reconduce el campo fenomenal a su autonomía, es decir, a un aparecer que, lejos de reposar en el elemento de la vivencia, se caracteriza por su función ostensiva o figurativa, es entonces auto-supresión en favor de lo trascendente, en pocas palabras, es constitutivamente aparecer de un mundo. En otras palabras, no existen datos hiléticos inmanentes que vendrían a animar la actividad noemática: la pretendida materia sensible es cada vez ya un momento mediador, según Patočka, que abre el objeto y es por tanto igualmente trascendente que él. La distinción pertinente no pasa más entre inmanencia y trascendencia sino entre una trascendencia subjetiva, aquella de los momentos mediadores correlativos de las coordenadas de un sujeto vivo, y una trascendencia objetiva, aquella de la cosa mediatizada por esos momentos. También podría decirse que el aparecer no es jamás exclusivamente inmanente sino al contrario siempre aparecer de un mundo. La residencia del aparecer en lo sucesivo se ha descentrado o, mejor dicho, se ha recentrado del lado del mundo. La fórmula a la cual hemos llegado -todo aparecer es aparecer del mundo- debe ser comprendida en un sentido esencial, es decir, como una caracterización de la esencia misma del aparecer. Ella significa que toda aparición se borra en favor de la trascen-

\footnotetext{
${ }^{6}$ Idem, p. 257.
} 
dencia mundana, es ostensión de dicha trascendencia, con lo cual es cada vez el mundo mismo que aparece en toda aparición. Patočka puede entonces resumir así la legalidad propia del aparecer: "Nosotros consideramos como perteneciente a la estructura del aparecer en cuanto tal esta totalidad universal del apareciente, el gran todo, así como aquello a lo cual el apareciente aparece, la subjetividad (teniendo una estructura pronominal vacía, que no debe identificarse con un sujeto singular cerrado)". ${ }^{7}$ La esencia misma del aparecer implica la referencia a este apareciente singular que es el mundo, lo que equivale a decir que toda aparición, en tanto que ella es verdaderamente una aparición (de otra cosa que de aquél a quién ella aparece) es necesariamente coaparición del mundo. Aquí, el contenido esencial de la aparición lo es en igual medida la forma, el apareciente último forma parte de la esencia del aparecer.

Esta primera conclusión exige dos consideraciones. En primer lugar, por supuesto ella no tiene sentido más que mediante una cierta acepción de mundo que ella contribuye a clarificar. El mundo no designa aquí evidentemente una totalidad aditiva de entes sino más bien aquello que excede el orden de lo óntico y puede así dirigir la aparición. El mundo es la totalidad universal, el "gran todo", esto es, la totalidad de todo aquello que puede aparecer, totalidad unitaria pero sin embargo abierta, que no puede entonces por principio aparecer como tal, porque solo se encuentra en toda aparición como lo que limita a dicha aparición, en pocas palabras, como el horizonte universal de toda aparición. Para decirlo de otra manera, se puede observar que si, la donación perceptiva del ente se basa en un decurso de perspectivas, la continuidad de la experiencia supone que me sea primero garantizado la posibilidad de proseguirla, que me sea entonces dado desde el principio su "continuabilidad" [continuabilité]. Como lo escribe Patočka, "que yo tenga siempre de nuevo, donde sea que me encuentre, la posibilidad de realizar la misma continuación, aquello no es simplemente anticipado, sino dado, bajo la forma, no de una simple intuición, sino de una presencia independiente del cumplimiento contingente o de la simple anticipación vacía". ${ }^{8}$ El mundo no es otra cosa que esta presencia englobante, esta escena para una continuación indefinida de la experiencia.

\footnotetext{
7 Patočka, J. (1995). Papiers phénoménologiques, trad. fr. E. Abrams, Grenoble, J. Millon, p. 177 [de igual manera, realizo una traducción personal del texto francés citado en el original].

${ }^{8}$ Idem, p. 179. Cf. p. 184: "Es verdad que aquello que está ausente no puede estar presente; pero el hecho que toda presencia sea parte de una sola y única presencia englobante puede, ella, estar presente; esta estructura, relativa a la totalidad, puede estar inmediatamente presente" [citado por Barbaras].
} 
Por otra parte, se sigue de este análisis una inversión pura y simple de la relación entre el ego y el mundo. Decir en efecto que el mundo es constitutivo de la estructura del aparecer, que toda aparición es por lo tanto co-aparición del mundo, viene a reafirmar que la aparición misma del ego se encuentra mediatizada por el mundo y que, en este sentido, el mundo es precisamente el a priori del ego, lejos de lo que pensaba Husserl que veía al ego como el a priori de la aparición del mundo. Esta inversión es el resultado inmediato de la epojé radicalizada mencionada más arriba: "Gracias a la universalización de la epojé, resultará evidente también que, del mismo modo que el yo es la condición de posibilidad del aparecer del mundo, igualmente el mundo como horizonte originario (y no como el conjunto de las realidades) representa la condición de posibilidad del aparecer del yo". ${ }^{9}$ De esta manera, el sí mismo no accede a él mismo más que a través de la mediación del mundo, no se encuentra a sí más que dejándose desposeer por el mundo, lo cual significa exactamente que él no se posee jamás, que él no puede por principio realizar la coincidencia. Decir en efecto que el yo no se encuentra a sí mismo más que en el mundo, pone de manifiesto en la misma medida que él está separado de sí mismo por la profundidad irreductible del mundo. En resumen, el yo y el mundo poseen unos estatutos exactamente paralelos, correspondientes a una apodicticidad sin adecuación posible; a semejanza del mundo, el yo está dado inmediatamente a sí mismo como aquello que por principio no puede estar presente. Sin embargo, tal conclusión permite asimismo transformar profundamente las coordenadas del problema. La verdadera cuestión ya no es más aquella del modo de donación del yo sino la de su sentido de ser en la medida en que aquél no puede encontrarse sino por la mediación del mundo. Sea como fuere, al término de este primer recorrido, que restituye eso que podríamos denominar la vertiente fenomenológica del pensamiento de Patočka, podemos afirmar que el sentido de la correlación fenomenológica ha sido profundamente modificado. Ella no debe ser más comprendida como constitución del mundo en una conciencia trascendental sino como relación entre un aparecer que es siempre del mundo, que descansa entonces de una cierta manera en él mismo y, por otra parte, un destinatario (el a quién del aparecer) que, si él es efectivamente co-

\footnotetext{
9 Patočka, J. Qu'est-ce que la phénoménologie?, p. 258 [traducción propia del texto referenciado en francés en el original].
} 
determinante, como lo dice Patočka, no es en ningún caso constituyente. Dicho de otro modo, si las apariciones se dan efectivamente a un sujeto, no es en virtud de dicha donación como ellas surgen como aparición de un mundo. Con ésta formulación nueva de la correlación, nos encontramos de inmediato confrontados a la más seria dificultad, aquella de comprender cómo puede haber un aparecer autónomo del mundo y, correlativamente, cómo determinar el estatuto exacto del destinatario de la aparición en esta economía del aparecer.

Pero antes de interrogarnos sobre su estatuto, nos es necesario abordar la cuestión de su sentido de ser, cuestión hacia la cual convergen todas nuestras consideraciones precedentes. Dicha cuestión nos permitirá pasar de la versión fenomenológica a la versión cosmológica del pensamiento de Patočka. La crítica patočkiana desemboca en un sujeto que ya no es el lugar y la condición del aparecer sino simplemente su destinatario, de forma que, en rigor, como lo veremos, un tal sujeto hace suyo y prolonga un aparecer del que no posee la iniciativa. Un tal sujeto pertenece al mundo al igual que todo ente, puesto que el mundo es la totalidad omni-englobante, pero distinguiéndose sin embargo de los otros entes en aquello que dicho sujeto hace aparecer el mundo o, en todo caso, se refiere a él en el modo de la percepción, de tal manera que con él los entes mundanos acceden al sentido. Nos encontramos aquí lo bastante cerca de la descripción heideggeriana del Dasein y no es por eso sorprendente que Patočka aborde la cuestión del sentido de ser del sujeto mediante una desformalización de la existencia del Dasein. Efectivamente, si Heidegger afirma por un lado la pertenencia del Dasein al mundo y su compromiso activo en él, por otra parte, él no integra en el seno de los existenciales del Dasein las condiciones de dicha pertenencia: "pareciera que la analítica vuelve la ontología heideggeriana de la existencia muy formal. La praxis es en efecto la forma original de claridad, pero Heidegger no toma jamás en consideración el hecho que la praxis original debe ser por principio la actividad de un sujeto corporal". ${ }^{10}$ Desde luego, como lo destaca Patočka, solo la corporeidad permite dar cuenta de la existencia y de su "en vista de" en su efectividad. Sin embargo, para ello, todavía falta superar la concepción aún ingenua del cuerpo como realidad sustancial ("presente aquí y ahora") y es sin duda por no haber percibido un sentido de ser del cuerpo

${ }^{10}$ Patočka, J. (1988). Le monde naturel et le mouvement de l'existence humaine, trad. E. Abrams, Kluwer, 1988, p. 93 [traducción propia del texto referenciado en francés en el original].

Investigaciones Fenomenológicas, n. 13, 2016. 
ajeno a la Vorhandenheit por lo que Heidegger no integra jamás el cuerpo en los existenciales del Dasein. En otras palabras, no resulta posible inscribir el cuerpo en el centro del Dasein a título de existencial sino con la condición de superar una caracterización sustancialista de dicho cuerpo en aras de una determinación existencial: el cuerpo no puede ser parte de la existencia más que si él es concebido existencialmente. Ahora bien, no puede ser ni el vector $y$, en realidad, ni el portador de una existencia que es por entero poder-ser, sino un cuerpo que es esencialmente (o más bien existencialmente) motricidad. Como lo escribe Patočka,

sin duda, todo aquello que realizo se hace con miras a mi ser, pero al mismo tiempo hay una posibilidad fundamental que me debe estar abierta, una posibilidad sin la cual todas las otras quedan suspendidas en el vacío, sin la cual ellas parecen desprovistas de sentido e irrealizables. [...] Esto significa que no se trata de una posibilidad entre otras, sino más bien de una posibilidad privilegiada que codeterminará en su sentido la existencia en su integridad. Esta base ontológica es la corporeidad como posibilidad de moverse. ${ }^{11}$

Así se justifica ante todo aquello que constituye sin lugar a dudas el núcleo del pensamiento de Patočka y su contribución más incontestable, a saber, la determinación de la existencia como movimiento. Esta determinación aparece finalmente como una síntesis entre la exigencia de encarnación del sujeto, puesta de relieve por Merleau-Ponty, y el enfoque existencial del Dasein abierto por Heidegger. El Dasein no puede estar verdaderamente en el mundo, es decir, actuar en él, más que bajo la condición de moverse en él y es a partir de dicha aptitud de moverse como se entiende la determinación más originaria de la corporeidad. Constatemos que, por el movimiento, el sujeto se distingue radicalmente de los otros entes, puesto que el movimiento no es en cierta manera sino una negación activa y efectiva de la entidad, aunque siga estando profundamente inscrito en el mundo y entonces en perfecta continuidad con los otros entes, en la medida en que no hay movimiento sino sobre el terreno del mundo.

Con todo, cabe destacar que esta determinación de la existencia a partir del movimiento se justifica también plenamente desde el punto de vista mismo de

${ }^{11}$ Idem, p. 96. 
la fórmula de la correlación a la cual hemos llegado. En efecto, el destinatario del aparecer es necesariamente un sujeto que es de alguna manera capaz de recuperar, de retomar por su cuenta el advenimiento de la aparición en tanto que esta es siempre co-aparición del mundo, apertura de una trascendencia pura. A la ostensión del mundo en una aparición debe por tanto responder un sujeto que es capaz de exceder la aparición en favor del mundo. No obstante, en la medida en que el mundo no es un ente sino una totalidad abierta, es decir, un puro exceso sobre el ente, el sujeto que corresponda a la aparición debe existir sobre un modo tal que sea capaz de igualarse a aquel puro exceso, de coincidir con la profundidad irreductible del mundo. Sin duda alguna, solo el movimiento responde a estas condiciones: a la trascendencia del mundo no puede corresponder más que el inacabamiento constitutivo del movimiento; el sujeto no puede alcanzar el mundo, condición última de toda aparición, más que tomando la delantera en él. Esa es la razón por la que Patočka define el sujeto como "fuerza vidente", "energía centrífuga" o incluso "impulso de penetración en el mundo", lo cual significa que el sujeto hace aparecer el mundo o, mejor dicho, participa en su aparición penetrando en él. Aquí, las consideraciones de inspiración heideggeriana sobre el sentido de ser del sujeto vienen a converger con las consideraciones de inspiración husserliana y merleaupontiana sobre la esencia de la percepción y la naturaleza necesariamente dinámica del sujeto perceptivo. Por cierto, queda por especificar la naturaleza exacta de aquel movimiento ya que él no puede ser identificado con un simple desplazamiento. Ahora bien, no es seguro que al hablar de movimientos generales de la existencia, distintos de su efectuación empírica necesariamente espacial, en un horizonte que sigue siendo al fin y al cabo heideggeriano, Patočka logre verdaderamente dar cuenta de la naturaleza exacta de aquel movimiento que es la existencia. Sea lo que sea, la crítica del subjetivismo y la reformulación de la correlación nos han conducido a aquello que podríamos denominar una fenomenología dinámica, en la medida que el sujeto (destinatario) del aparecer se encuentra allí caracterizado como movimiento. Sin embargo, sigue siendo preciso franquear un paso más.

Al insistir en la autonomía del aparecer, Patočka quiere resaltar el hecho de que el aparecer del mundo no es la obra de una conciencia, que las vivencias no son el elemento del aparecer: si el sujeto forma parte del aparecer del mundo, él no lo constituye de manera alguna. De este modo, haciendo hincapié en 
la dimensión propiamente dinámica del sujeto, Patočka muestra que el sujeto es desposeído por el mundo más que éste sea poseído por él, y que es ésta su manera propia de relacionarse con él. No obstante, tal conclusión no puede quedar sin incidencia alguna sobre el sentido de ser del mundo mismo y conduce en realidad a superar el ámbito en el cual nos hemos situado inicialmente. En efecto, si existe realmente una autonomía del aparecer, si verdaderamente toda aparición efectúa por ella misma la exhibición del mundo, de manera que la actividad propia del sujeto no consiste sino en acompañar por así decirlo dicha efectuación, entonces es necesario concluir que es el mundo mismo el que aparece en toda aparición, en el sentido que dicho aparecer es su obra propia. La afirmación según la cual toda aparición es aparición del mundo debe entonces ser comprendida en los dos sentidos del genitivo: desde luego, toda aparición empieza con el mundo, lo desvela, pero esto es en la medida exacta en que es el mundo mismo quien está en el origen de su aparición, dándole así lugar. El mundo no parece en toda aparición sino siempre y cuando él se haga ser en ella: toda aparición es el revés de un advenimiento y de una producción. Entonces ya no podemos atenernos a la caracterización aún abstracta del mundo como totalidad. Ésta no tiene valor sino desde un punto de vista que es todavía subjetivista, ella no recupera el mundo más que en y desde su aparecer, como aquella totalidad que se da en toda aparición. Pero, desde el instante en que examinamos el mundo en sí mismo, como aquello cuya aparición no depende de ningún sujeto, es decir, como el artífice de su propia aparición, nos encontramos obligados a ir más allá de la idea del mundo como totalidad en favor de una caracterización de naturaleza dinámica. Remarquemos, sin embargo, en seguida que esta aproximación, que inclina la balanza del aparecer del lado del mundo mismo, vuelve de súbito extraordinariamente problemático el surgimiento de aquello que hasta ahora era evidente, en tanto que zócalo del aparecer, a saber, el sujeto mismo.

Esta modificación del sentido de ser del mundo que se impone sobre la base de una concepción a-subjetiva del aparecer puede ser confirmada a partir de la fenomenología dinámica. En efecto, hemos señalado que el sujeto del aparecer pertenecía necesariamente al mundo, no obstante, se hace necesario añadir ahora que el despliegue del movimiento sobre la base del mundo no responde plenamente a esta pertenencia. Ésta última posee una significación ontológica y no solamente espacial: dicho de otro modo, si el sujeto puede desplazarse en el 
seno del mundo y contribuir a su aparición, es en la medida exacta en que dispone de un parentesco ontológico con él, en que él es del mundo, como decía Merleau-Ponty. Tan sólo esta afirmación de una pertenencia cuya significación es en última instancia ontológica no deja de tener repercusiones sobre el sentido de ser que debe concederse al mundo. Si, como lo hemos establecido, el sujeto es movimiento y pertenece al mundo bajo el modo de un parentesco ontológico, entonces el ser del mundo debe él mismo estar situado del lado del movimiento, el enfoque estático del mundo que prevalecía hasta ahora debe ser superado en beneficio de un enfoque decididamente dinámico. Nuestro movimiento como tal se despliega sin duda sobre el telón de fondo del mundo que es totalidad, pero el sujeto de dicho movimiento está en continuidad con un mundo que es en el fondo una realidad procesual, de modo que el movimiento del sujeto proviene en realidad del proceso mismo del mundo. Así, la diferencia del sujeto con respecto del mundo como totalidad de entes no se opone a una identidad más profunda con el mundo entendido como realidad dinámica. Patočka había percibido perfectamente esta consecuencia ontológica de la caracterización dinámica del sujeto. En una carta inédita a Robert Campbell del 20 de marzo de 1964, él escribió: "El devenir y el movimiento que está en el origen de todas nuestras experiencias es él mismo imposible sin un devenir más profundo y más elemental que es, no movimiento en la experiencia y en el mundo, sino devenir y movimiento del mundo como tal, devenir ontológico". A esta observación hace eco un texto publicado que esboza este nuevo enfoque del mundo: "en tanto que nosotros nos movemos, en tanto que actuamos y que, en este "hacer", nos comprendemos a nosotros mismos, así como las cosas, nosotros formamos parte de la physis, del mundo omni-englobante, de la naturaleza". ${ }^{12}$

También podría decirse que la fenomenología dinámica, por la que el ser del sujeto debe ser comprendido como movimiento, se supera hacia lo que podríamos denominar una dinámica fenomenológica. Para ésta, la fenomenalización misma se debe entender como movimiento, lo cual significa que la esencia del movimiento es la de recubrir una fenomenalización. Ahora bien, este pasaje de una fenomenología dinámica a una dinámica fenomenológica también lo es del pasaje de una fenomenología a una cosmología, puesto que dicho pasaje

${ }^{12}$ Idem, p. 107.

| Investigaciones Fenomenológicas, n. 13, 2016. 
significa que la fuente y el sujeto primero del aparecer debe ser buscado en una physis. En otras palabras, el movimiento subjetivo, la fuerza vidente se inscriben en un proceso de fenomenalización más profundo, proceso anónimo que ellos vienen, en cierta medida, a prolongar recogiéndolo. El movimiento que llevamos a cabo refleja pues un concepto más originario de movimiento: "Concept de movimiento como fundamento -movimiento concebido, no como movimiento del objeto, sino como obra de la physis antes de cualquier objetivación o subjetivación- la physis como esencia que es acontecimiento, esencia que ocurre". ${ }^{13}$ No podemos decirlo mejor, el movimiento que está en el origen del aparecer corresponde a un sentido inédito del movimiento, puesto que no se refiere a un sustrato en particular sino, al contrario, a la condición de emergencia de todo sustrato. Extranjero al orden de lo óntico dado que él es la condición de emergencia, tal movimiento es asimismo indiferente a la dicotomía de lo subjetivo y lo objetivo. Finalmente, y por eso mismo, este movimiento no tiene esencia ni se remite a esencia alguna puesto que es el movimiento mismo del advenimiento de la esencia. Mostraríamos sin dificultades, pero nos falta el espacio para hacerlo, que aquel movimiento debe ser comprendido como un proceso de individuación, es decir, como una salida de la indiferenciación. Este proceso de aparición es un proceso de delimitación de los entes: él nombra el nacimiento mismo de lo múltiple a partir de la noche de la indiferenciación y, por consiguiente, de la totalidad como unidad de aquel múltiple. De lo anterior se desprende en primer lugar la exigencia de distinguir al menos tres sentidos del mundo. Por supuesto, como ya lo hemos visto, el mundo como totalidad de los entes debe ser reconducido al mundo fenomenológico como horizonte último, esto es, como totalidad abierta que no existe fuera de lo que ella totaliza, e incluso como el entramado [Ajointement ${ }^{14}$ en tanto tal. Pero el mundo fenomenológico debe él mismo ser reconducido a la physis siempre que dicha totalidad del horizonte se sumerja en la unicidad del proceso mundificante de la que ella es por así decirlo la traza. En el seno de lo múltiple, ella es la potencia misma del fondo que se sedimenta como totalidad. Por otra parte, es en la medida en que la manifestación debe ser en última instancia comprendida como levantamiento de una ocultación que es originariamente indiferenciación, como

\footnotetext{
${ }^{13}$ Patočka, J. Papiers phénoménologiques, p. 269 [traducción propia del texto referenciado en francés en el original].

${ }^{14}$ Traduce al francés la palabra alemana Gefüge. [Nota de la redacción].
} 
el proceso mundificante-diferenciante puede ser identificado con un proceso de ma-nifestación. Como lo escribe Patočka, "cada cosa adquiere su figura - se delimita- deviene en tanto que individuo poniéndose aparte de los otros. Este devenir traza unas fronteras con respecto de otras cosas, él es un proceso de definición, de puesta en forma. Esta definición se hace respecto de todas las demás cosas, y todas son co-definidas. Este definir -el aparecer de la cosa en cuanto su ser". ${ }^{15}$ Así, en la medida en que la ocultación se confunde con la noche de la indiferenciación, el surgimiento del ente por delimitación, es decir, su individuación se confunde ipso facto con su aparición.

Hemos ido con Patočka lo más lejos posible en la crítica del subjetivismo y a su vez en la tentativa de descripción de una fenomenalización que sea autónoma, independiente de la actividad de un sujeto. En efecto, con esta caracterización dinámica del mundo hemos encontrado un proceso que es por entero y eminentemente movimiento, puesto que él hace ser los entes singulares, y es por derecho propio fenomenalización en la medida en que dicha delimitación es sinónimo de desocultación. Nos es preciso entonces asumir la conclusión, a primera vista sorprendente, según la cual nos estamos enfrentado aquí a una manifestación que no es todavía manifestación a alguien, una manifestación sin sujeto, anónima. Asimismo, esta manifestación es la condición verdadera de la manifestación subjetiva, del aparecer a, ya que el movimiento que hace advenir los entes a sus sentidos procede de aquel que hace nacer los entes mismos, y que la síntesis de identificación presupone una síntesis que ocurre en las cosas mismas, una síntesis material. ${ }^{16}$ Esto es lo que Patočka reconoce por sí mismo haciendo un guiño a Fink cuando éste habla de una manifestación que "no es manifestación para el sujeto, sino más bien manifestación como ingreso en la singularidad, devenir. Se trata de una manifestación en la que las cosas singularizadas son ellas mismas interiormente indiferentes, una manifestación que no es manifiesta a ella misma, una manifestación inmersa en la obscuridad nocturna del ente y del ser originarios". ${ }^{17}$ Así, el punto de culminación de aquel movimiento de desubjetivación del aparecer es la distinción entre dos grados de la manifestación, o incluso dos tipos de apertura. Existe la manifestación primaria y anónima correspondiente al archi-movimiento de mundificación y la mani-

${ }^{15}$ Idem, p. 114. Cf. también Patočka, J. Platon et l'Europe, p. 169 [referencia en francés en el original].

${ }^{16}$ Cf. Patočka, J. Papiers phénoménologiques, p. 32.

17 Patočka, J. Le monde naturel et le mouvement de l'existence humaine, p. 100. [traducción propia del texto referenciado en francés en el original].

Investigaciones Fenomenológicas, n. 13, 2016. 
festación secundaria como manifestación a un sujeto reposando sobre su propio movimiento. E incluso, en los términos que son aquellos del mismo Patočka, existe la apertura primordial, "física" del ente por el proceso mundano y la apertura secundaria del ente por el sujeto.

Es en este punto donde nos encontramos expuestos a la dificultad más aguda, que es la contrapartida del camino que hemos recorrido hasta ahora. Efectivamente, llevada hasta sus últimas consecuencias, la crítica del subjetivismo obliga a reconocer un aparecer que es la obra misma del mundo y cuya esencia no parece implicar la referencia a un sujeto. Pero entonces aquello que era evidente, a saber, la existencia del sujeto, es lo que plantea de repente un problema. Por supuesto, no se trata en absoluto de poner en tela de juicio la existencia del sujeto y de abandonar el marco de la correlación. Es innegable que el archi-movimiento del mundo da origen a nuestro movimiento, es decir, al sujeto que nosotros somos; resulta poco discutible que la delimitación producida por el archi-movimiento esté destinada a ser identificada como unidad de sentido para un sujeto. La cuestión es más bien aquella de la relación entre los dos niveles del aparecer y entonces entre las dos modalidades de movimientos, del modo de surgimiento del sujeto al interior del proceso mundano, en pocas palabras, la cuestión es aquella de la articulación entre el plano fenomenológico -aquel del aparecer a un sujeto- y el plano cosmológico del aparecer "en sí". Pues bien, cada vez que se tropieza con esta cuestión, Patočka manifiesta un desconcierto evidente puesto que, por un lado, no puede renunciar a las adquisiciones de la desubjetivación y por tanto a la autonomía del aparecer, pero, por otro, no quiere sacrificar el nivel propiamente subjetivo que garantiza el marco fenomenológico. La vía en la que se compromete es entonces aquella de una prefiguración del aparecer subjetivo en el aparecer primario, de modo que, finalmente, el aparecer subjetivo es reconsiderado como el telos del aparecer primario, nuestro movimiento como el telos del archi-movimiento del mundo. Así, Patočka llega a escribir que existe una relación entre el aparecer a mí y el fondo mismo del mundo, siendo aquél una dimensión del mundo y concluye: "Así se encuentra enraizado en el fondo mismo del mundo un ente que no es necesario, pero que hace necesariamente que lo necesario emergente delante de él aparezca como un modo de ser deficiente, algo que, claro, aparece y que 
es, pero al cual le falta la claridad del compromiso [l'intéressement] consigo mismo, el relacionar-se interiormente en sí mismo y con el otro" ${ }^{18} \mathrm{~A}$ pesar del carácter embarazoso de la frase, la referencia a la falta, incluso si tiene validez solo desde el punto de vista del sujeto, no deja lugar a dudas: el aparecer primario está caracterizado por un defecto de ser respecto al aparecer subjetivo, que es su cumplimiento mismo.

No podemos dejar de pensar que comprometiéndose en esta dirección Patočka pierde lo esencial de lo que había adquirido y no queda finalmente redimido del subjetivismo al que se dedica, sin embargo, a cuestionar sin cesar. El sujeto sigue siendo en efecto la residencia del aparecer a título de su telos; el aparecer sigue siendo esencialmente aparecer a un sujeto, pero teleológicamente y no constitutivamente. Resumiendo, es siempre el horizonte de la subjetivación el que controla la descripción del aparecer, de manera que la distancia de Patočka frente al subjetivismo no corresponde sino a la brecha que existe entre un sujeto constituyente y un sujeto que funciona como fin. En un sentido, Patočka hegelianiza a Husserl, pero sin ir más allá. Ahora bien, tal solución no es en ningún caso satisfactoria porque entra en conflicto con las consecuencias más evidentes del pasaje efectuado hacia la cosmología. De hecho, a nivel del archi-movimiento del mundo, nada nos permite comprender que éste da origen a un sujeto, es decir, a un individuo que, contrariamente a los otros, se encuentre en posición de relacionarse con los otros y con la totalidad. Y es porque se da cuenta de que Patočka será conducido a optar por la solución de la teleología, solución que no es realmente una, puesto que proyecta el resultado en el origen a falta de lograr fundarlo en la naturaleza del proceso originario. Puesto que no hay sujetos y puesto que nada en el archi-movimiento permite comprender su nacimiento, tenemos que suponer que éste tiende hacia ellos. Con todo, el error de Patočka es quedarse encerrado en el marco de la cosmología que él mismo instituyó, y buscar allí un camino de acceso al sujeto que no se encuentra en la misma.

Existe otra solución, que es absolutamente fiel a la autonomía del aparecer y que equivale, como siempre, a determinar la gravedad del problema. Ella consiste en reconocer que nada, en el proceso de la manifestación primaria, que a su vez es aquél de la esencia, anuncia la posibilidad de un sujeto si bien

${ }^{18}$ Idem, p. 269.

Investigaciones Fenomenológicas, n. 13, 2016. 
existe un aparecer secundario, subjetivo. La conclusión se nos impone: el advenimiento del sujeto no puede proceder más que de algo que afecta al archimovimiento mundano sin pertenecerle y que se remite a un plano que escapa tanto a la fenomenología como a la cosmología. Ahora bien, ¿cómo denominar aquello que le ocurre al movimiento sin estar, de ninguna manera, incluido en él, que es por consiguiente absolutamente imprevisible, sino como un acontecimiento [événement]? El sujeto procede entonces de un archi-acontecimiento que viene a afectar y modificar al archi-movimiento del mundo. Lejos entonces de estar premeditado en el archi-movimiento de la manifestación, el sujeto es lo imprevisible mismo; lejos de ser el zócalo o el telos de toda esencia, él es al contrario lo sin razón por excelencia. Por otra parte, ¿qué le puede suceder a este archi-movimiento que todo lo produce y que podríamos definir como archivida? ¿Qué le puede ocurrir a aquello por lo cual todo ocurre? Precisamente nada, sino es bajo la forma efectiva de una escisión o de una separación, única figura posible de la negatividad en el seno de la potencia del archi-movimiento. Es necesario entonces decir que todo sucede como si el archi-movimiento del mundo se hubiera desprendido o se hubiera separado de sí mismo, se hubiera caído fuera de sí mismo, en resumen, como si estuviese afectado por esta negatividad pura que es la separación en cuanto que ella no es nada más que aquello que ella separa. Este archi-acontecimiento corresponde finalmente a la diferencia mínima requerida por la correlación en el marco cosmológico continuista del archi-movimiento: no hay dos términos o dos centros sino un solo movimiento; sin embargo, hay correlación porque dicho movimiento se ve afectado por un archi-acontecimiento. Este archi-acontecimiento puede entonces ser descrito como advenimiento de una segunda individuación, por separación o por exilio y ya no más por delimitación. Él es advenimiento de lo viviente en el seno de la archi-vida, es decir, igualmente advenimiento de la mortalidad. En efecto, separada de ella misma en un viviente, la potencia de la archi-vida está destinada a agotarse y el sujeto viviente a regresar a la archi-vida dejando de estar afectado por el archi-acontecimiento.

Esta condición es aquella de la subjetividad misma y el descubrimiento de dicha dimensión nueva permite aclarar el estatuto de este movimiento subjetivo que Patočka ha descubierto. El paso decisivo, que Patočka no traspasa, aquél que lo conduce a una versión teleológica de la unidad de los dos niveles del aparecer, consiste en reconocer que, lejos de ser un modo de cumplimiento 
del proceso de manifestación, la subjetivación es al contrario la consecuencia de un defecto de ser, de una debilidad [faiblesse]. Es en la medida exacta en que el archi-movimiento del mundo pierde algo de sí mismo, o más bien cae fuera de sí mismo, como se hace movimiento subjetivo. Es en la medida en que la individuación productiva del mundo se prolonga en una existencia exiliada como ella deviene identificación. Por consiguiente, el ente viviente individualizado por separación se caracteriza por la impotencia, siendo sin embargo en él donde la potencia del mundo se agota. Pero esta impotencia tiene como revés una cierta potencia, aquella de hacer parecer. En efecto, mientras que el movimiento del mundo se refiere a los entes sobre el modo del producir, el sujeto viviente no puede relacionarse con ellos sino sobre el modo del deseo: separado del movimiento del mundo del que procede su ser, él no puede sino aspirar a dicho ser. Es en este deseo en el que consiste precisamente el movimiento subjetivo, que Patočka denomina movimiento de la existencia. Deseando el ente a falta de poder producirlo, el sujeto libera el principio de su unidad, es decir, el sentido; a falta de hacerlo ser, él lo hace parecer. El sentido corresponde a un defecto de ser, a aquello que queda del ente cuando un sujeto se relaciona sin poder producirlo. El sentido es el estricto correlato del deseo. Así, a favor del archi-acontecimiento que separa el proceso mundificante de sí mismo, el movimiento impotente del deseo puede surgir $y$, con él, una unidad separada del ente de la que ella es la unidad.

Finalmente, habrán ya comprendido, este archi-acontecimiento atañe a lo que nosotros denominaremos una metafísica y es por tanto con la condición de superar la cosmología misma, lo que Patočka no hace, como podremos verdaderamente fundar una fenomenología no-subjetivista. El archi-acontecimiento atañe a la metafísica en tanto que escapa al orden de la esencia, éste último siendo el del archi-movimiento. Ella se refiere al sentido husserliano de la metafísica como descubrimiento de una archi-facticidad de la que no hay ni esencia, ni causa. Esta metafísica, que se hace cargo del archi-acontecimiento en su imprevisibilidad, al mismo tiempo que de sus consecuencias, se encuentra en las antípodas de la metafísica de la subjetividad, que subyace aún en el pensamiento del mismo Patočka: ella es metafísica de la imposibilidad de la subjetividad. Es a la exploración de esta metafísica a la que debe consagrarse, según nosotros, una fenomenología fiel a sus propios principios.

(Traducción de Manfredi Moreno)

| Investigaciones Fenomenológicas, n. 13, 2016. 